\title{
CDKN2B-AS1 Genotype - Glaucoma Feature Correlations in Primary Open-Angle Glaucoma Patients from the United States
}

Louis R. Pasquale(1),(2), Stephanie J. Loomis(1), Jae H. Kang ${ }^{(2)}$, Brian L. Yaspan(3), ${ }^{*}$, Wael Abdrabou $^{(1)}$, Donald L. Budenz ${ }^{(4)}$, Teresa C. Chen ${ }^{(1)}$, Elizabeth DelBono(1), David S. Friedman $^{(5)}$, Douglas Gaasterland ${ }^{(6)}$, Terry Gaasterland ${ }^{(7)}$, Cynthia L. Grosskreutz ${ }^{(1)}$,

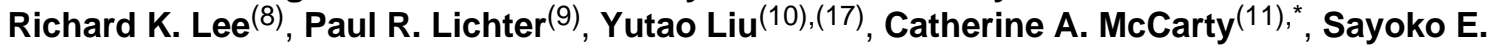
Moroi $^{(9)}$, Lana M. Olson ${ }^{(3)}$, Tony Realini ${ }^{(12)}$, Douglas J. Rhee(1), Joel S. Schuman ${ }^{(13)}$, Kuldev Singh $^{(14)}$, Douglas Vollrath ${ }^{(15)}$, Gadi Wollstein ${ }^{(13)}$, Donald J. Zack ${ }^{(5)}$, R. Rand Allingham ${ }^{(10)}$, Margaret A. Pericak-Vance $^{(8)}$, Robert N. Weinreb ${ }^{(16)}$, Kang Zhang ${ }^{(16)}$, Michael A. Hauser ${ }^{(10)}$, (17), Julia E. Richards ${ }^{(9),(18)}$, Jonathan L. Haines ${ }^{(3)}$, and Janey L. Wiggs ${ }^{(1)}$

(1)Department of Ophthalmology, Mass Eye \& Ear Infirmary, Harvard Medical School, Boston, MA

(2)Department of Medicine, Brigham and Women's Hospital, Harvard Medical School, Boston, MA

(C) 2012 Elsevier Inc. All rights reserved.

Correspondence and reprints: Louis R. Pasquale, MD; Mass Eye and Ear Infirmary; 243 Charles Street; Boston, MA 02114; (TEL): 617-573-3674; (FAX) 617-573-4300; Louis_Pasquale@meei.harvard.edu.

* Dr. McCarty is now at Essentia Institute of Rural Health, Duluth, MI and Dr. Yaspan is now at Genentech, Inc, San Francisco, CA. Supplemental Material available at AJO.com

Author contributions: Design and conduct of the study (LRP, SJL, JHK, RKL, RRA, JER, JLW); All authors contributed to the collection of data. Management of the data (SJL, JHK, BLY, ED); Analysis and interpretation of the data (LRP, SJL, JHK, RRA, JLW); Review of the manuscript (LRP, SJL, JHK, BLY, WA, DLB, TC, ED, DG, CG, RKL, PRL, YL, CAM, SEM, LO, TR, DJR, KS, GW, RRA, RNW, MAH, JER, JLW).

Financial disclosures: Dr. Pasquale has received funding from Merck for unrelated investigator initiated-research. He was also reimbursed for travel to ARVO-Pfizer meeting on biomarkers in glaucoma research in May 2011. Dr. T Gaasterland has received an honorarium for a lecture given on glaucoma genomics by Sequenom. Dr. Yaspan contributed to this research while he was at Vanderbilt University and is currently an employee of Genentech. He has no other financial conflicts to declare. Dr. Grosskreutz is an employee of Novartis and retains stock options from her employer. She has no other financial conflicts to declare.

The following coauthors declare the following financial activities for work outside the scope of the current publication. Dr. Budenz is a consultant to Alcon Labs, Allergan, Santen, Ivantis and Alimera. His institution receives grant support from Carl Zeiss Meditec and New World Medical. Dr. Budenz is on the speakers bureau for Merck, Alcon and Allergan. Dr. Chen receives grant support from the Harvard Catalyst group, the National Institutes of Health (NIH), and the Agency for Healthcare Research. She also receives royalties from Elsevier for her role as a book editor. Dr. Friedman is a consultant for Alcon, Bausch and Lomb, Merck, Pfizer and QLT. Dr. D. Gaasterland has been paid royalties as inventor of the G probe for Iridex. He also owns a small amount of IRIDEX stock. Dr. Lee receives research support from National Eye Institute (NEI) and the American Glaucoma Society. He is also on the speakers bureaus of Merck and Pfizer. Dr. Lee receives royalties from Pharmigen and has been reimbursed for meeting travel by Alcon. Dr. Realini is a consultant for Alcon and received payment for manuscript preparation from this company. He is also on the speakers bureau for Lumenis. Dr. Morio receives grant support from Merck and NEI as well as royalties from Lippicott. Dr. Rhee is a consultant for Alcon, Allergan, Merck, Novagali, and Santen. He also receives grant support from Alcon and Merck. Dr. Schuman receives grant support from the NIH and is an inventor on a patent (paid to University of Pittsburgh). He receives royalties from Carl Zeiss Meditec. Dr. Singh is consultant to Alcon, Allergan, Santen, Bausch and Lomb. Dr. Wollstein receives grant support from NIH. Drs. Allingham and Pericak-Vance receive grant support from NIH. Dr. Zack retains Board membership with Alcon and has received grant support form Merck. Dr. Weinreb is a consultant to Alcon, Allergan, Merck, Bausch \& Lomb, Zeis Meditec and Optovue. He also receives grant support form Novartis, Nidek, Topcon, and Aeries. Dr. Richards receives grant support from NIH, Research to Prevent Blindness, and the Sramek Foundation. She also receives royalties for authoring a textbook from Elsevier and has been reimbursed for travel to a Think Tank meeting by the Glaucoma Foundation, NYC. The following coauthors have no financial disclosures to declare: Ms. Loomis, Dr. JH Kang, Mr. Abdrabou, Ms. Delbono, Dr. Lichter, Dr. McCarty, Ms. Olson, Dr. Vollrath, Dr. Hauser, Dr. K. Zhang, Dr. Haines and Dr. Wiggs.

Publisher's Disclaimer: This is a PDF file of an unedited manuscript that has been accepted for publication. As a service to our customers we are providing this early version of the manuscript. The manuscript will undergo copyediting, typesetting, and review of the resulting proof before it is published in its final citable form. Please note that during the production process errors may be discovered which could affect the content, and all legal disclaimers that apply to the journal pertain. 
${ }^{(3)}$ Center for Human Genetics Research, Vanderbilt University, Nashville, TN

(4)Department of Ophthalmology, University of North Carolina, Chapel Hill, NC

${ }^{(5)}$ Wilmer Eye Institute, Johns Hopkins University Hospital, Baltimore, MD

(6)Eye Doctors of Washington, Chevy Chase, MD

(7)Scripps Genome Center, University of California at San Diego, San Diego, CA

${ }^{(8) B a s c o m ~ P a l m e r ~ E y e ~ I n s t i t u t e ~ a n d ~ H u m a n ~ G e n o m i c s, ~ U n i v e r s i t y ~ o f ~ M i a m i ~ M i l l e r ~ S c h o o l ~ o f ~}$ Medicine, Miami, FL

(9)Department of Ophthalmology and Visual Sciences, University of Michigan, Ann Arbor, MI

${ }^{(10)}$ Department of Ophthalmology, Duke University Medical Center, Durham, NC

(11)Center for Human Genetics, Marshfield Clinic Research Foundation, Marshfield, WI

${ }^{(12)}$ Department of Ophthalmology, West Virginia University Eye Institute, Morgantown, WV

(13)Department of Ophthalmology, UPMC Eye Center, University of Pittsburgh, Pittsburgh, PA

(14)Department of Ophthalmology, Stanford University, Palo Alto, CA

(15)Department of Genetics, Stanford University, Palo Alto, CA

(16)Department of Ophthalmology and Hamilton Glaucoma Center, University of California, San Diego, La Jolla, CA

(17)Department of Medicine, Duke University Medical Center, Durham, NC

${ }^{(18)}$ Department of Epidemiology, University of Michigan, Ann Arbor, MI

\section{Abstract}

PURPOSE-To assess the association between single nucleotide polymorphisms (SNPs) of the gene region containing cyclin dependent kinase inhibitor 2B antisense noncoding RNA $(C D K N 2 B-A S 1)$ and glaucoma features among primary open-angle glaucoma (POAG) patients.

DESIGN-Retrospective observational case series.

METHODS-We studied associations between ten CDKN2B-AS1 SNPs and glaucoma features among 976 POAG cases from the Glaucoma Genes and Environment (GLAUGEN) study and 1971 cases from the National Eye Institute Glaucoma Human Genetics Collaboration (NEIGHBOR) consortium. For each patient, we chose the feature from the eye with the higher value. We created cohort-specific multivariable models for glaucoma features and then metaanalyzed the results.

RESULTS-For nine of the ten protective $C D K N 2 B-A S 1$ SNPs with minor alleles associated with reduced disease risk (e.g., the $\mathrm{G}$ allele at rs2157719), POAG patients carrying these minor alleles had smaller cup-disc ratio (0.05 units smaller per $\mathrm{G}$ allele at diagnosis; $95 \% \mathrm{CI}$ : -0.08 , -0.03 ; $p=6.23 \mathrm{E}-05)$ despite having higher intraocular pressure (IOP) $(0.70 \mathrm{~mm} \mathrm{Hg}$ higher per $\mathrm{G}$ allele at DNA collection; 95\% CI: 0.40, 1.00; P=5.45E-06). For the one adverse rs3217992 SNP with minor allele A associated with increased disease risk, POAG patients with A alleles had larger cup-disc ratio (0.05 units larger per A allele at diagnosis; 95\% CI: 0.02, 0.07; P=4.74E-04) despite having lower IOP $(-0.57 \mathrm{~mm} \mathrm{Hg}$ per A allele at DNA collection; 95\% CI: $-0.84,-0.29$; $\mathrm{P}=6.55 \mathrm{E}-05)$.

CONCLUSION-Alleles of $C D K N 2 B-A S 1$ SNPs, which influence risk of developing POAG, also modulate optic nerve degeneration among POAG patients, underscoring the role of CDKN2B-AS1 in POAG. 


\section{INTRODUCTION}

The cyclin dependent kinase inhibitor 2B antisense noncoding RNA (CDKN2B-ASI) genomic region on chromosome $9 \mathrm{p} 21.3$ is a genetic susceptibility locus for several agerelated complex diseases (for an explanation of genetic terminology used in this manuscript see Table 1). ${ }^{1}$ Genome-wide association (GWA) studies and candidate gene investigations indicate that gene variants in this region are associated with primary open-angle glaucoma (POAG) but the relation between $C D K N 2 B-A S 1$ genetic variants and specific glaucoma features are not well known. ${ }^{2-7} C D K N 2 B-A S 1$ is an antisense RNA that may influence the nearby $C D K N 2 A$ (cyclin dependent kinase inhibitor $2 \mathrm{~A}$ ) and $C D K N 2 B$ (cyclin dependent kinase inhibitor 2B) genes via regulatory mechanisms. ${ }^{8} C D K N 2 A$ and $C D K N 2 B$, which are expressed in all cell types, influence cell proliferation ${ }^{9,10}$ and senescence. ${ }^{11-14}$ Retinal ganglion cells (RGCs), a target for degeneration in POAG, must maintain a quiescent postmitotic state for an indefinite period in order to carry out their physiologic functions. In POAG, elevated intraocular pressure (IOP) and other insults may trigger quiescent RGCs to undergo apoptosis. ${ }^{15,16}$

Sequence variants in $C D K N 2 B-A S 1$ are associated with cup-disc ratio in a normal population. ${ }^{17}$ Interestingly, common minor alleles for several of these variants are associated with a smaller cup-disc ratio in normal subjects, ${ }^{17}$ as well as a reduced risk of POAG.$^{2-7}$ Cup-disc ratio is a structural optic nerve feature highly correlated with the POAG disease process. POAG is a form of deleterious optic nerve aging without obvious secondary cause that is exacerbated by intraocular pressure (IOP) and ultimately results in functional visual loss.

In this study we used the data from POAG cases enrolled in two large case-control groups where GWA studies have been completed: the GLAUGEN (Glaucoma Genes and Environment) study, part of the GENEVA (the Gene, Environment Association Studies) consortium ${ }^{18}$ and the NEIGHBOR (National Eye Institute Glaucoma Human Genetics Collaboration) consortium. ${ }^{19}$ In order to gain further insight regarding the $C D K N 2 B-A S 1$ genomic region and the glaucomatous process, we assessed the association between ten $C D K N 2 B-A S 1$ single nucleotide polymorphisms (SNPs) strongly associated with POAG and glaucoma features like age at diagnosis, IOP parameters, cup-disc ratio, visual field parameters, and the need for laser trabeculoplasty (LTP) or incisional surgery.

\section{METHODS}

The Institutional Review Boards of Mass Eye and Ear Infirmary, Brigham and Women's Hospital, Harvard School of Public Health as well as the Institutional Review Boards of the participating NEIGHBOR institutions prospectively approved the genotyping efforts, data analyses and data sharing arrangements described herein. Each participant gave written consent to participate in this observational case series.

\section{Description of the study populations}

The GLAUGEN study consists of POAG cases and controls drawn from the Nurses' Health Study (NHS), the Health Professionals Follow-up Study (HPFS) and the Genetic Etiologies of Primary Open-Angle Glaucoma study (GEP). The former two studies are populationbased, nested case-control studies and the latter study is a clinic-based, case-control study from the Massachusetts Eye and Ear Infirmary (MEEI). Details regarding the inclusion/ exclusion criteria for the GLAUGEN POAG case-control cohort have been described (www.ncbi.nlm.nih.gov/projects/gap/cgi-bin/study.cgi?study_id=phs000308.v1.p1). 
The NEIGHBOR consortium consists of POAG cases and controls from twelve sites. Details regarding the study sites, design, inclusion criteria and clinical variables collected in the NEIGHBOR consortium have also been described elsewhere. ${ }^{19}$

\section{Case definition}

All cases had slit lamp examinations, which did not reveal secondary cause of elevated IOP (such as exfoliation syndrome) and the anterior chamber angle was deemed non-occludable. We did not employ IOP criteria in defining POAG. All cases had either reproducible visual field (VF) loss or one abnormal VF associated with cup-disc ratio $>0.7$ in the eye with loss. Reproducible VF loss consistent with nerve fiber layer pathology had to be demonstrated on tests that were considered reliable (fixation loss $\_33 \%$, false positive rate $\_20 \%$ and false negative rate $\mathbf{2} 0 \%$ ). We categorized the observed VF loss depending on whether it involved the paracentral zone, nasal step region, Bjerrum's area and the temporal wedge region above and/or below the horizontal meridian based on a systemic evaluation of the pattern deviation plot or its equivalent. There was no restriction placed on the type of VF perimeter used.

\section{Genotyping data}

Details regarding DNA collection, extraction, and plating for GLAUGEN ${ }^{20}$ and NEIGHBOR ${ }^{5}$ have been previously described. We used the Illumina Human660W-Quad-v1 array (Illumina; San Diego, CA) for high throughput genotyping. Both sets of genotyping data were subject to extensive quality control checks that have been previously described. 5,20

\section{Gene association analyses and the 9p21 polymorphisms chosen for analysis}

After employing quality control filters, gene association analyses in GLAUGEN and NEIGHBOR were performed using PLINK v1.07. ${ }^{21}$ Details regarding these analyses have been described elsewhere ${ }^{5,20}$ Briefly, in GLAUGEN among 976 cases and 1140 controls, no genetic loci achieved genome-wide significant association with POAG. In NEIGHBOR, among 2170 cases and 2347 controls, 17 SNPs reached genome-wide significance (p SE-08), 16 of which were in the $C D K N 2 B-A S 1$ region. ${ }^{5}$ The top SNP associated with POAG was rs4977756 (Odds Ratio=0.66; 95\% CI: 0.59-0.73; $\mathrm{p}=7.4 \mathrm{E}-16$ ). Using METAL, we performed a meta-analysis of the GLAUGEN and NEIGHBOR dataset and identified 19 SNPs with genome-wide significant associations in relation to POAG, and 17 of these were in the $C D K N 2 B-A S 1$ region. In this study of POAG phenotypes, we chose to analyze the top ten $C D K N 2 B-A S 1$ SNPs associated with POAG in the meta-analysis. The effect sizes, $\mathrm{p}$-values and risk alleles for the chosen SNPs in relation to POAG are provided in the supplemental Table.

\section{Ascertainment of glaucoma phenotype features}

We required that all cases included in our study have data on age at diagnosis (Table 2). It can be difficult to ascertain age at diagnosis for POAG, as it is an insidious onset disease; yet, the determination of whether $C D K N 2 B-A S 1$ variants are associated with this glaucoma feature is important because an earlier age of diagnosis could translate into more visual disability from the condition later in life. Age at diagnosis in GLAUGEN and in NEIGHBOR was defined as the age at first sign of disease, namely, cup-disc ratio $>0.6$, cupdisc ratio asymmetry $\searrow 0.2$, IOP $>21 \mathrm{~mm} \mathrm{Hg}$, or VF loss which was ascertained based on medical record review.

The IOP recorded was either at the time of diagnosis or at DNA collection (which was typically after diagnosis and while under treatment especially for NEIGHBOR participants). 
We also recorded whether there was a history of IOP > $21 \mathrm{~mm} \mathrm{Hg}$ or the highest known IOP when such data was available. Similarly, cup-disc ratio recorded was either at diagnosis or at DNA collection. The type of IOP and cup-disc ratio data available varied by study subtype. Specifically, IOP and cup-disc ratio at diagnosis were available for NHS and HPFS cases in GLAUGEN. We excluded all NEIGHBOR cases from the assessment of $C D K N 2 A B-A S 1$ SNPs in relation to IOP and cup-disc ratio at diagnosis as continuous variables because these features were only available in the Collaborative Initial Glaucoma Treatment Study (www.clinicaltrials.gov; NCT00000149) site. Nonetheless, a history of IOP $\geq 22 \mathrm{~mm} \mathrm{Hg}$ at diagnosis was available on all NEIGHBOR participants. IOP and cup-disc ratio at DNA collection were available among MEEI cases in GLAUGEN and all NEIGHBOR cases except the Johns Hopkins University (JHU) and CIGTS sites. At the JHU site of NEIGHBOR, the collected IOPs represent the highest known values. For all study sites, the higher values between eyes were chosen for analysis. Box plots depicting the distribution of IOPs and cup-disc ratio by study site are provided in Supplemental Figures 1 and 2. Median IOP was $>21 \mathrm{~mm} \mathrm{Hg}$ in NHS, HPFS, CIGTS, the Advanced Glaucoma Intervention Study (AGIS; www.clinicaltrials.gov; NCT00000148) site and the JHU cases. For all other sites, the median IOP ranged between 15 and $18 \mathrm{~mm} \mathrm{Hg}$. Median cup-disc ratios ranged between 0.6-0.7 for sites with incident cases (NHS and HPFS in GLAUGEN and CIGTS in NEIGHBOR) and 0.8-0.9 across study centers with mostly prevalent cases (MEEI in GLAUGEN and West Virginia University, University of Pittsburgh, JHU, Stanford University, Duke University, University of Michigan, AGIS and Marshfield Clinic in NEIGHBOR). Since age at diagnosis was unavailable at the University of Miami site, these cases were excluded from all $C D K N 2 B-A S 1$ - glaucoma feature analyses.

For the VF global indices, mean defect (MD) and pattern standard deviation (PSD), we chose the parameter from the eye showing more functional loss on the earliest available Humphrey VF test. A glaucoma specialist (LRP) reviewed all VF tests and excluded cases judged to have non-glaucomatous loss (such as age-related macular degeneration) or lens rim artifact from $C D K N 2 B-A S 1$ genotype - correlations analysis with MD, PSD and pattern of VF loss. Furthermore, because PSD begins to decline with severe generalized reduction of retinal sensitivity, ${ }^{22}$ we excluded patients with MD values worse than $-13 \mathrm{~dB}$ in the analysis of the relation between $C D K N 2 B-A S 1$ SNPs and PSD (47 exclusions in GLAUGEN and 238 in NEIGHBOR). This cutoff was chosen based on inspection of a graph of MD vs. PSD using the earliest available VF from NEIGHBOR and GLAUGEN participants (data not shown).

VFs were classified as having 'peripheral VF loss only' if the paracentral zone was not involved. In contrast, VFs were categorized as having 'paracentral VF loss only' if the nasal step regions, Bjerrum areas and temporal wedge areas were not involved. Furthermore, VFs were classified as having 'superior VF loss only' if the inferior hemifield of the pattern deviation plot was normal. The opposite was true for VF with 'inferior VF loss only'. We also classified patients with 'paracentral and peripheral loss' and patients with 'superior and inferior loss.'

In GLAUGEN we collected data on a history of LTP and incisional glaucoma surgery of any kind. In NEIGHBOR this data was only available on from the CIGTS and the AGIS sites. Since these procedures were performed as part of randomized clinical trials in CIGTS ${ }^{23}$ and $\mathrm{AGIS}^{24}$, these data on laser and incisional surgery were excluded from genotype-phenotype correlations.

\section{Statistical analysis}

We created multivariable linear regression models for continuous phenotypes and multiple logistic regression models for categorical phenotypes using SAS9.2 (Cary, NC). CDKN2B- 
AS1 SNPs were coded as minor allele dose variables with 3 values: $0=$ no minor allele, $1=1$ minor allele, and $2=2$ minor alleles. We first analyzed the data from GLAUGEN and NEIGHBOR separately and performed tests for heterogeneity to check for appropriateness of pooling the results. When appropriate, we pooled the results using meta-analytic methods. ${ }^{25} \mathrm{We}$ adjusted for variables that influenced genotyping call rates (which were all $>98 \%$ in both studies): DNA source (blood or cheek), study site (NHS, HPFS or GEP in GLAUGEN or the study subsite in NEIGHBOR), and the method of DNA extraction (GENTRA, DNAzol or QIAGEN). We also adjusted the relation between genotypes and glaucoma features for population structure (a genetic surrogate of ancestry) using 3 eigenvectors in GLAUGEN and 2 eigenvectors in NEIGHBOR. Finally we also adjusted for age at diagnosis (expect when this was the outcome of interest) and gender in all models. We used the Bonferroni correction, accounting for the number of unique linkage disequilibrium (LD) blocks where our SNPs were located (five blocks; see Supplemental Figure 3) and the number of unique glaucoma features we assessed as outcomes (ten), to establish p-values $<0.001$ as statistically significant.

In secondary analyses assessing the association of $C D K N 2 B-A S 1$ SNPs with CDR parameters, we additionally controlled for atonal homolog 7 ( $A$ TOH7) SNPs (rs7916697 and rs 3858145 ) strongly correlated with disc area. ${ }^{17,26}$ For associations between $C D K N 2 B$ $A S 1$ SNPs that showed statistically significant associations with VF parameters, we further adjusted for cup-disc ratio. Finally, since age at diagnosis can depend on IOP level but IOP criteria were not employed in diagnosing glaucoma, we performed a secondary analysis of the relation between $C D K N 2 B-A S 1$ and age at diagnosis among cases with no history of IOP $\geq 22 \mathrm{~mm} \mathrm{Hg}$.

\section{RESULTS}

The 976 GLAUGEN patients and 1971 NEIGHBOR patients for whom we recorded the age at diagnosis represents $100 \%$ and $90.8 \%$ of the POAG cases respectively that completed high throughput genotyping in these cohorts. Tables 2 and 3 summarize the demographic and ocular features for POAG cases. The mean age at diagnosis was slightly less in GLAUGEN than in NEIGHBOR. The minimal age at diagnosis in these studies corresponds to the minimal age criteria for inclusion in the respective studies. As expected, mean IOP at diagnosis was higher than the mean IOP at DNA collection because the former represented untreated levels (Table 2). Similarly, the CDR at diagnosis was smaller than the cup-disc ratio at DNA collection because the latter came from prevalent cases. While VF loss was required for all cases, MD values were only available for $87 \%$ and $69 \%$ of GLAUGEN and NEIGHBOR cases respectively because not all subjects had Humphrey VFs. Table 3 also shows the percent of patients with the various types of VF loss patterns.

The LD block structure for the $C D K N 2 B-A S 1$ SNPs is provided in Supplemental Figure 3. The minor allele for the most upstream SNP (rs32177992) is associated with increased POAG risk; the remainder are associated with decreased POAG risk. ${ }^{5}$

Table 4 provides data for the associations between each minor allele for $C D K N 2 B-A S 1$ SNPs and continuous glaucoma features after meta-analysis of the GLAUGEN and NEIGHBOR data. Table 5 shows the associations between each minor allele for the $C D K N 2 B-A S 1$ SNPs and dichotomous glaucoma features determined by meta-analysis.

\section{9p21 polymorphisms in relation to age at diagnosis / intraocular pressure variables in primary open-angle glaucoma cases}

There was no significant association between any $C D K N 2 B-A S 1$ SNP and age at glaucoma diagnosis ( $\mathrm{n}=2,947$ cases; Table 4). In secondary analysis limited to cases with IOP $<22$ 
mm Hg ( $\mathrm{n}=573$ cases), one SNP (rs573687) was significantly associated with a later age at diagnosis after accounting for the multiple comparisons made. This SNP was associated with a reduced risk of POAG in our case-control study. Each minor allele (A) in this SNP was associated with developing disease 4.5 years later $(95 \% \mathrm{CI}: 2.04,10.1$ years; $\mathrm{p}=0.0002)$ than people who were homozygous for the reference genotype (GG). There was no significant association between any $C D K N 2 B-A S 1$ SNPs and IOP at diagnosis $(\mathrm{n}=345$ cases; Table 4). However, there were associations between $C D K N 2 B-A S 1$ SNPs and a history of IOP $>21 \mathrm{~mm} \mathrm{Hg}$ at diagnosis, which was available for a larger group of cases $(n=2,195$; Table 5). For example, for rs1063192, which is inversely related to POAG, there was a $39 \%$ increased risk of IOP > $21 \mathrm{~mm} \mathrm{Hg}$ at diagnosis $(95 \% \mathrm{CI}: 1.17,1.66$; $\mathrm{p}=2.00 \mathrm{E}-04$ ) per minor allele (Table 5). Similar trends were seen for three other SNPs that were associated with reduced risk of POAG. On the other hand, for each additional minor allele of rs3217992, which is associated with increased POAG risk, IOP at DNA collection was $0.57 \mathrm{~mm} \mathrm{Hg}$ lower per minor allele (95\% CI: $-0.84,-0.29 \mathrm{~mm} \mathrm{Hg}$; $=6.55 \mathrm{E}-05)$ (Table $4 ; \mathrm{n}=1,942$ cases). The associations between $C D K N 2 B-A S 1$ SNPs and IOP at DNA collection remained after excluding cases with a history of incisional glaucoma surgery ( $\mathrm{p} \leq 1.61 \mathrm{E}-04$; data not shown).

\section{9p21 polymorphisms in relation to cup-disc ratio variables}

POAG cases with protective $C D K N 2 B-A S 1$ alleles tended to have smaller cup-disc ratio at diagnosis (0.05-0.06 units lower per minor allele; $\mathrm{p} \leq 1.96 \mathrm{E}-04 ; \mathrm{n}=458)$ than cases homozygous for the common reference genotypes (Table 4). Conversely, cases with minor alleles for the adverse SNP (rs32177992), had larger cup-disc ratio at diagnosis (Table 4). The statistically significant results remained even after adjustment for SNPs associated with disc size in $A T O H 7$ (p $\ 3.85 \mathrm{E}-04$; data not shown). Similar trends were observed when cupdisc ratio at diagnosis was treated as a dichotomous variable $(>0.6$ or $\$) .6$ ) but the results were not significant after adjusting for multiple comparisons (Table 5). There were no associations between $C D K N 2 B-A S 1$ SNPs and cup-disc ratio at the time of DNA collection using either linear regression (Table 4) or logistic regression (Table 5) models.

\section{9p21 polymorphisms in relation to visual field features}

We found no significant relation between $C D K N 2 B-A S 1$ SNPs and MD from the earliest Humphrey VFs using multivariable linear regression analysis (Table 4). However, among cases with MD better than $-13 \mathrm{~dB}$, there were significant relationships between $C D K N 2 B$ $A S 1$ SNPs and PSD. For the SNP associated with increased POAG risk (rs3217992), each minor allele was associated with a $0.50 \mathrm{~dB}$ higher PSD $(95 \% \mathrm{CI}: 0.30 \mathrm{~dB}, 0.71 \mathrm{~dB}$; $\mathrm{p}=1.50 \mathrm{E}-06$ ). This association was essentially unchanged when additionally controlled for cup-disc ratio at diagnosis in NHS and HPFS and cup-disc ratio at DNA collection for all other participants (0.46 dB higher; $95 \% \mathrm{CI}$ : $0.24 \mathrm{~dB}, 0.68 \mathrm{~dB}$; $\mathrm{p}=5.35 \mathrm{E}-05)$. For the three significant SNPs associated with decreased POAG risk (rs7049105, rs2151280 and rs1012068), each minor allele was associated with a 0.35-0.36 dB lower PSD (p $₫ .00 \mathrm{E}-04$; Table 4). While cases with minor alleles of selected protective $C D K N 2 B-A S 1$ SNPs had lower PSD, these latter associations were not significant after controlling for cup-disc ratio.

Interestingly, for each minor allele of rs3217992 (which was associated with increased POAG risk), there was a $21 \%$ reduced risk of 'peripheral VF loss only' (95\% CI: 0.70, 0.89; $\mathrm{p}=1.00 \mathrm{E}-04)$. Conversely, for each minor allele of the protective SNP rs2157719, there was a $24 \%$ increased risk of 'peripheral VF loss only' in one or both eyes (95\% CI: 1.09-1.41; $\mathrm{p}=9.00 \mathrm{E}-04)$. These associations were not significant after controlling for cup-disc ratio. No other significant associations between $C D K N 2 B-A S 1$ SNPs and pattern of VF loss were detected. 


\section{9p21 in relation to a history of laser trabeculoplasty and incisional glaucoma surgery}

With data available on 976 GLAUGEN cases, we found no association between $C D K N 2 B$ $A S 1$ SNPs and a history of incisional glaucoma surgery among POAG cases (Table 5). Similarly we did not find associations between $C D K N 2 B-A S 1$ SNPs and a history of LTP among POAG cases (data not shown).

\section{DISCUSSION}

POAG cases with the minor alleles in rs3217992 (which increases POAG risk) had larger cup-disc ratio at diagnosis and a higher PSD on the earliest available VF manifesting functional loss despite having lower IOP at DNA collection. In contrast, cases with minor alleles in selected SNPs that reduce POAG risk had smaller cup-disc ratio at diagnosis and increased chance of peripheral VF loss only on the earliest VF despite an increased IOP at DNA collection. These data suggest that genotypes in the $C D K N 2 B-A S 1$ region modulate vulnerability to glaucoma. It is not surprising that POAG patients would harbor protective $C D K N 2 B-A S 1$ variants, as they are common among Caucasians and POAG is a polygenetic disease modified by environmental influences. ${ }^{27,28}$

\section{Age at diagnosis and history of incisional glaucoma surgery}

We observed no significant associations between $C D K N 2 B-A S 1$ SNP genotypes and age at glaucoma diagnosis $(\mathrm{n}=2,947)$. Limiting analysis to cases where there was no known IOP $\geq$ $22 \mathrm{~mm} \mathrm{Hg}$ ( $\mathrm{n}=573$ cases), there was a SNP associated with a later age at diagnosis. As we learn more about the genetic architecture of POAG, knowledge of genotypes that influence age of disease onset among people with normal IOP and glaucoma-like discs may play a role in optimizing glaucoma management. We found no relation between $C D K N 2 B-A S 1 \mathrm{SNP}$ genotype and history of incisional surgery among GLAUGEN cases. Only 15\% of 976 cases in this group required glaucoma filtration surgery, which might have limited the power to detect modest associations between $C D K N 2 B-A S 1$ SNPs and this glaucoma feature. Furthermore the reasons to undergo surgery for this group of patients are heterogeneous and likely transcend issues related solely to recalcitrance to medical therapy and LTP. Nonetheless, this study suggests that $C D K N 2 B-A S 1$ genotypes seem unrelated to a history of incisional glaucoma surgery, a feature suggestive of increased risk of vision loss via exposure to potential surgical complications.

\section{Intraocular pressure variables}

$C D K N 2 B-A S 1$ SNPs were significantly associated with IOP at DNA collection in 1,942 cases, even though IOP levels at DNA collection were influenced by glaucoma treatment. For example, POAG patients possessing protective $C D K N 2 B-A S 1$ minor alleles had higher IOP at DNA collection than patients who were homozygous for the corresponding more common reference genotypes. Similar associations were observed between these same alleles and IOP at diagnosis but the results were not statistically significant ( $\mathrm{n}=345$ cases) perhaps reflecting the smaller sample size. The similar effect estimates for IOP at diagnosis and at DNA collection probably reflect a correlation between untreated and treated IOP. ${ }^{29}$ In contrast, POAG patients harboring the minor alleles in rs3217992 that predispose to disease had lower IOP at DNA collection than patients who were homozygous for the more common reference genotype. These data suggest that $C D K N 2 B-A S 1$ SNPs modulate susceptibility to IOP. There is already some data suggesting this effect could be generalized to other glaucomas, as Wiggs et al. found rs2157719 is also associated with reduced risk of exfoliation syndrome-related open angle glaucoma but not exfoliation syndrome alone. ${ }^{5}$ 


\section{Cup-disc ratio and visual field parameters}

$C D K N 2 B$ - $A S 1$ SNPs were originally described in association with cup-disc ratio, ${ }^{17}$ raising the question of whether this genomic region merely explains variance in normal optic nerve structure or whether this region is associated with optic nerve pathology that is responsible for the visual consequences seen in glaucoma. Variants protective against POAG were associated with smaller cup-disc ratio at disease presentation while the opposite was true for the adverse variant. Some of the same protective variants were also associated with smaller cup-disc ratio in a large population from Rotterdam, most of which did not have glaucoma. ${ }^{17}$

Because there is some correlation between cup-disc ratio (structure) and PSD (function) ( $\mathrm{R}=0.18 ; \mathrm{p} \unlhd 0.0001$ in GLAUGEN), we purposefully imposed an additional term for cupdisc ratio in our model to assess the relation between $C D K N 2 B-A S 1$ SNPs and PSD. While most of the associations between $C D K N 2 B-A S 1$ SNPs and PSD became insignificant, the significant association between rs3217992 and PSD remained, suggesting this SNP renders the optic nerve susceptible to focal nerve fiber layer dropout in POAG. Thus it seems plausible that $C D K N 2 B-A S 1$ variants explain both normal and pathologic changes in optic nerve structure that produce functional consequences.

Finding genetic variants linked to incident paracentral visual loss is of utmost importance because this pattern of loss places POAG patients at higher risk of developing visual disability as the disease advances. We found no association between $C D K N 2 B-A S 1$ allelic variants and paracentral VF loss ( $\mathrm{n}=214$ cases with paracentral VF loss only). However, we observed that the adverse SNP variants in rs3217992 were associated with a reduced risk of peripheral VF loss only ( $\mathrm{n}=950$ cases). Conversely, the protective variants in rs 2157719 were associated with an increased chance of peripheral VF loss only. The significance of these statistical associations was attenuated when cup-disc ratio was additionally controlled for; however, it is unclear if this represents a form of over controlling. It is possible that our inability to find associations with paracentral VF loss could be due to lack of power, given that only $\sim 18 \%$ in GLAUGEN and 3\% in NEIGHBOR had this glaucoma feature.

The unique strength of this study is its large sample size with 9p21 region genotypes and glaucoma feature data available on up to 2,947 POAG cases from throughout the United States. Shortcomings of the study include the fact that glaucoma feature information was collected in a non-uniform manner and was missing for some cases. While this study was limited entirely to Caucasians, it is interesting to inspect the minor allele frequency (MAF) of the 9p21 gene region variants in the African population from Yoruba. The MAF of $C D K N 2 B-A S 1$ SNPs associated with smaller cup-disc ratio are generally much higher in Caucasians than in African people (Supplemental Table) (www.ncbi.nlm.nih.gov/SNP; dbSNP Build ID:37.3). For example, the allelic variant of rs2157719 which is associated with reduced risk of $\mathrm{POAG}^{5}$ and a smaller CDR in POAG cases, has a MAF of 0.44 in Caucasians but is rare in a African population from Nigeria (MAF=0). Perhaps the relative low mean allele frequencies of protective $C D K N 2 B-A S 1$ alleles in African populations overall explains the results of a recent study that showed normal Caucasians had more favorable PSD values than normal African Americans on Humphrey VF tests. ${ }^{30}$ Several studies also find larger cup-disc ratio in African people but this difference could reflect the presence of a larger disc area. ${ }^{30-34}$ Interestingly, Cao et al. reported that the protective relation between the minor allele in rs 1063192 and POAG found in Caucasians ${ }^{3,5}$ is also noted in an Afro-Caribbean population. ${ }^{35}$ The authors postulate that population admixture may have increased the allele frequency of the minor allele for this SNP relative to subSaharan Africans ${ }^{35}$ where this allele is virtually non-existent (see supplemental Table). 
In conclusion, in this US-based observational case study, we found associations between $9 p 21$ variants and glaucoma features that suggest this region modifies optic nerve vulnerability to glaucomatous change. In fact our CDKN2B-AS1 - glaucoma feature correlations agree with the study published by Burdon et al. ${ }^{36}$ Our study provides further support for the notion that the $C D K N 2 B-A S 1$ region is important for maintaining RGCs in a stable post-mitotic state. These data underscore the need to determine how $C D K N 2 B-A S 1$ region gene variants impact RGC biology. As the genetic landscape for POAG becomes clearer, knowledge of 9 p21 region genotypes may be useful for optimizing glaucoma management.

\section{Supplementary Material}

Refer to Web version on PubMed Central for supplementary material.

\section{Acknowledgments}

Funding/Support: A Horizon Grant to MEEI from Allergan (Irvine, CA) supported the collection of some glaucoma feature data. The Harvard Glaucoma Center of Excellence and Margolis fund (Boston, MA) support WA, LRP and JLW. LRP, JER and JLW are also supported by Research to Prevent Blindness, Inc. (New York, NY). The Glaucoma Research Foundation (San Francisco, CA), American Health Assistance Foundation (Clarksburg, MD), and the Glaucoma Foundation (New York, NY) support YL.

The following National Institutes of Health grants support the maintenance of the Nurses Health Study and Health Professionals Follow-up, allowing these health professionals to contribute to this analysis: CA87969, CA49449, CA55075, HL35464, and NEI R01 EY015473 (LRP).

The following grants from the National Human Genome Research Institute (Bethesda, MD) supported GLAUGEN: HG004728 (LRP), HG004424 (Broad Institute to support genotyping), HG004446 (C. Laurie, U. Washington, to support genotype data cleaning and analysis).

Genotyping services for the NEIGHBOR study were provided by the Center for Inherited Disease Research (CIDR) and were supported by the National Eye Institute through grant HG005259-01 (JLW). Additionally CIDR is funded through a federal contract from the National Institutes of Health to The Johns Hopkins University, contract number HHSN268200782096C. The National Eye Institute (Bethesda, MD) through ARRA grants 3R01EY015872-05S1 (JLW) and 3R01EY019126-02S1 (MAH) supported the collection and processing of samples for the NEIGHBOR dataset.

Funding for the collection of cases and controls was provided by National Institutes of Health (Bethesda, MD) grants: EY015543 (RRA), EY006827 (DG), HL073389 (Hauser, E); EY13315 (MAH); EY09611 (Hankinson, S), EY009149 (PRL, HG004608 (CAM), EY008208 (Medeiros, P.), EY015473 (LRP), EY012118 (MAP-V), EY015682 (TR), EY011671 (JER), EY09580 (JER), EY013178 (JSS), EY015872 (JLW), EY010886 (JLW), EY009847 (JLW), EY011008 (Zangwill, L), EY144428 (KZ), EY144448 (KZ), EY18660 (KZ).

Other acknowledgements: The authors acknowledge Mr. Daniel Pasquale of Mass Eye and Ear Infirmary who assisted in collecting glaucoma feature data. We used the following web resources in the conduct of this study: PLINK (http://pngu.mgh.harvard.edu/ purcell/plink/ and METAL (http://www.sph.umich.edu/csg/Metal/ download)

\section{References}

1. Pasmant E, Sabbagh A, Vidaud M, Bieche I. ANRIL, a long, noncoding RNA, is an unexpected major hotspot in GWAS. FASEB J. 2011; 25(2):444-448. [PubMed: 20956613]

2. Burdon KP, Macgregor S, Hewitt AW, et al. Genome-wide association study identifies susceptibility loci for open angle glaucoma at TMCO1 and CDKN2B-AS1. Nat Genet. 2011; 43(6): 574-578. [PubMed: 21532571]

3. Fan BJ, Wang DY, Pasquale LR, Haines JL, Wiggs JL. Genetic variants associated with optic nerve vertical cup-to-disc ratio are risk factors for primary open angle glaucoma in a US Caucasian population. Invest Ophthalmol Vis Sci. Mar; 2011 52(3):1788-1792. [PubMed: 21398277] 
4. Ramdas WD, van Koolwijk LM, Lemij HG, et al. Common genetic variants associated with openangle glaucoma. Hum Mol Genet. 2011; 20(12):2464-2471. [PubMed: 21427129]

5. Wiggs J, Yaspan B, Hauser M, et al. Common variants in the CDKN2BAS region on 9p21 and a novel region on $8 \mathrm{q} 23$ are associated with the normal tension form of open-angle glaucoma with increased susceptibility to optic nerve degeneration. PLoS Genetics. 2012; 8(4):e1002654. [PubMed: 22570617]

6. Nakano M, Ikeda Y, Tokuda Y, et al. Common variants in CDKN2B-AS1 associated with optic nerve vulnerability of glaucoma identified by genome-wide association studies in Japanese. PLoS One. 2012; 7(3):e33389. [PubMed: 22428042]

7. Osman W, Low SK, Takahashi A, Kubo M, Nakamura Y. A genome-wide association study in the Japanese population confirms 9p21 and 14q23 as susceptibility loci for primary open angle glaucoma. Human Molecular Genet. 2012; 21(12):2836-42.

8. Cunnington MS, Santibanez Koref M, Mayosi BM, Burn J, Keavney B. Chromosome 9p21 SNPs Associated with Multiple Disease Phenotypes Correlate with ANRIL Expression. PLoS Genet. 2010; 6(4):e1000899. [PubMed: 20386740]

9. Schoppmeyer K, Norris PS, Haas M. Inhibition of T-cell acute lymphoblastic leukemia proliferation in vivo by re-expression of the p16INK4a tumor suppressor gene. Neoplasia. 1999; 1(2):128-137. [PubMed: 10933047]

10. Latres E, Malumbres M, Sotillo R, et al. Limited overlapping roles of P15(INK4b) and P18(INK4c) cell cycle inhibitors in proliferation and tumorigenesis. The EMBO Journal. 2000; 19(13):3496-3506. [PubMed: 10880462]

11. Loughran O, Malliri A, Owens D, et al. Association of CDKN2A/p16INK4A with human head and neck keratinocyte replicative senescence: relationship of dysfunction to immortality and neoplasia. Oncogene. 1996; 13(3):561-568. [PubMed: 8760298]

12. Erickson S, Sangfelt O, Heyman M, Castro J, Einhorn S, Grander D. Involvement of the Ink4 proteins p16 and p15 in T-lymphocyte senescence. Oncogene. 1998; 17(5):595-602. [PubMed: 9704925]

13. McConnell BB, Starborg M, Brookes S, Peters G. Inhibitors of cyclin-dependent kinases induce features of replicative senescence in early passage human diploid fibroblasts. Curr Biol. 1998; 8(6):351-354. [PubMed: 9512419]

14. Fuxe J, Akusjarvi G, Goike HM, Roos G, Collins VP, Pettersson RF. Adenovirus-mediated overexpression of p15INK4B inhibits human glioma cell growth, induces replicative senescence, and inhibits telomerase activity similarly to p16INK4A. Cell Growth Differ. 2000; 11(7):373-384. [PubMed: 10939591]

15. Wax MB, Tezel G, Edward PD. Clinical and ocular histopathological findings in a patient with normal-pressure glaucoma. Arch Ophthalmol. 1998; 116(8):993-1001. [PubMed: 9715678]

16. Kerrigan LA, Zack DJ, Quigley HA, Smith SD, Pease ME. TUNEL-positive ganglion cells in human primary open-angle glaucoma. Arch Ophthalmol. 1997; 115(8):1031-1035. [PubMed: 9258226]

17. Ramdas WD, van Koolwijk LM, Ikram MK, et al. A genome-wide association study of optic disc parameters. PLoS Genet. 2010; 6(6):e1000978. [PubMed: 20548946]

18. Cornelis MC, Agrawal A, Cole JW, et al. The Gene, Environment Association Studies consortium (GENEVA): maximizing the knowledge obtained from GWAS by collaboration across studies of multiple conditions. Genet Epidemiol. 2010; 34(4):364-3721. [PubMed: 20091798]

19. Wiggs J, Hauser M, Abdrabou W, et al. The NEIGHBOR Consortium Primary Open Angle Glaucoma Genome-wide Association Study: Rationale, study design and clinical variables. J Glaucoma. 2012 forthcoming.

20. Wiggs JL, Hee Kang J, Yaspan BL, et al. Common variants near CAV1 and CAV2 are associated with primary open-angle glaucoma in Caucasians from the USA. Hum Mol Genet. 2011; 20(23): 4707-4713. [PubMed: 21873608]

21. Purcell S, Neale B, Todd-Brown K, et al. PLINK: a tool set for whole-genome association and population-based linkage analyses. Am J Hum Genet. 2007; 81(3):559-575. [PubMed: 17701901]

22. Blumenthal EZ, Sapir-Pichhadze R. Misleading statistical calculations in far-advanced glaucomatous visual field loss. Ophthalmology. 2003; 110(1):196-200. [PubMed: 12511366] 
23. Musch DC, Lichter PR, Guire KE, Standardi CL. The Collaborative Initial Glaucoma Treatment Study: study design, methods, and baseline characteristics of enrolled patients. Ophthalmology. 1999; 106(4):653-662. [PubMed: 10201583]

24. The Advanced Glaucoma Intervention Study (AGIS): 1. Study design and methods and baseline characteristics of study patients. Controlled Clinical Trials. 1994; 15(4):299-325. [PubMed: 7956270]

25. DerSimonian R, Laird N. Meta-Analysis in Clinical Trials. Controlled Clinical Trials. 1986; 7:177-188. [PubMed: 3802833]

26. Macgregor S, Hewitt AW, Hysi PG, et al. Genome-wide association identifies ATOH7 as a major gene determining human optic disc size. Hum Mol Genet. 2010; 19(13):2716-2724. [PubMed: 20395239]

27. Fingert JH. Primary open-angle glaucoma genes. Eye (Lond). 2011; 25(5):587-595. [PubMed: 21562585]

28. Liu Y, Allingham RR. Molecular genetics in glaucoma. Exp Eye Res. 2011; 93(4):331-339. [PubMed: 21871452]

29. Leffler CT, Amini L. Interpretation of uniocular and binocular trials of glaucoma medications: an observational case series. BMC Ophthalmology. 2007; 7:17. [PubMed: 17916260]

30. Girkin CA, Sample PA, Liebmann JM, et al. African Descent and Glaucoma Evaluation Study (ADAGES): II. Ancestry differences in optic disc, retinal nerve fiber layer, and macular structure in healthy subjects. Arch Ophthalmol. 2010; 128(5):541-550. [PubMed: 20457974]

31. Beck RW, Messner DK, Musch DC, Martonyi CL, Lichter PR. Is there a racial difference in physiologic cup size? Ophthalmology. 1985; 92(7):873-876. [PubMed: 4022570]

32. Chi T, Ritch R, Stickler D, Pitman B, Tsai C, Hsieh FY. Racial differences in optic nerve head parameters. Arch Ophthalmol. 1989; 107(6):836-839. [PubMed: 2730402]

33. Tsai CS, Zangwill L, Gonzalez C, et al. Ethnic differences in optic nerve head topography. J Glaucoma. 1995; 4(4):248-257. [PubMed: 19920682]

34. Varma R, Tielsch JM, Quigley HA, et al. Race-, age-, gender-, and refractive error-related differences in the normal optic disc. Arch Ophthalmol. 1994; 112(8):1068-1076. [PubMed: 8053821]

35. Cao D, Jiao X, Liu X, et al. CDKN2B Polymorphism Is Associated with Primary Open-Angle Glaucoma (POAG) in the Afro-Caribbean Population of Barbados, West Indies. PLoS One. 2012; 7(6):e39278. [PubMed: 22761751]

36. Burdon KP, Crawford A, Casson RJ, et al. Glaucoma Risk Alleles at CDKN2B-AS1 Are Associated with Lower Intraocular Pressure, Normal-Tension Glaucoma, and Advanced Glaucoma. Ophthalmology. 2012 Apr 23.:1-7. [Epub ahead of print]. 10.1016/j.ophtha. 2012.02.004 
Table 1

Explanation of genetic terminology used in this manuscript:

Allele -one member of a DNA sequence pair that contributes to a trait. DNA sequence is comprised of a string of the following bases: adenine [A], guanine [G], cytosine [C] or thymine [T].

Antisense noncoding RNA - a single-strand RNA that does not result in protein translation; rather it can bind to nearby genes to alter their expression.

CDKN2BAS - cyclin dependent kinase inhibitor 2B antisense RNA - a segment of DNA that generates noncoding RNA located near the CDKN2B gene on chromosome 9.

Eigenvector - as it relates to an assessment of population structure, these are mathematically derived vectors representing a set of high throughput genotypes into clusters that reflect ancestral tendencies.

Genotype - specifies the pair of alleles (1 paternal and 1 maternal) at any specific genomic location; for example the following genotypes are possible at rs3217792 for $C D K N 2 B A S$ : GG (wild type), GA, AA (homozygous genotype for the minor variant).

Genome wide association (GWA) study - a study of the relation between a series of gene variants strategically located throughout the genome and a trait of interest (such as primary open-angle glaucoma).

Illumina Human660W-Quad-v1 array - a type of commercially available chip that allows for genotyping at 660,000 locations throughout the human genome on a DNA sample.

Linkage disequilibrium block (LD block)- a region in the genome where a set of SNPs is non-randomly associated with each other. When the genotype of one SNP in an LD block is known, the genotypes of the other SNPs in the block can be predicted with reasonable accuracy.

METAL - a contraction of meta analysis, refers to a statistical software tool to synthesis large datasets such as high throughout genotype data from various sources in a computer memory efficient manner. For more information about METAL see www.sph.umich.edu/csg/abecasis/ Metal/

Minor allele frequency (MAF)/minor allele - refers to the frequency of occurrence of the less common allele (aka, the minor allele) when single nucleotide differences exist between members of the same species. For example two individuals may contain the following sequence variants: CGAACTA and CGAATTA. The underlined nucleotide represents a single nucleotide polymorphic site and if $\mathrm{T}$ is less common and occurs $15 \%$ of the time, we say the MAF for T is 0.15 .

PLINK - an open-source analysis toolset used to analyze high throughput genotyping data. For more information about PLINK see http:// pngu.mgh.harvard.edu/ purcell/plink/

rs numbers (also known as ref SNP numbers) - an assignment or address for a polymorphic site in the human genome. For example, rs3217792 resides in the CDKN2BAS region on chromosome 9p21. The major allele at this site is G present in $\sim 63 \%$ of Caucasians and the minor allele at this site is A present in $37 \%$ of Caucasians.

SNP-single nucleotide polymorphism - a substitute of one allele for another in the genome; these substitutions can be common or rare and may or may not effect the function of the genomic segment in question. A common working hypothesis in genetic epidemiology research is that common SNPs serve as risk factors for complex diseases that are not inherited in a Mendelian fashion. Consider three unrelated individuals with a hypothetical stretch of DNA sequence:

--ATATCCG-

--ATATCCG-

--GTATCCG-

The first base represents a polymorphic site or SNP in that the A allele is changed to a G allele for the third DNA sequence. 

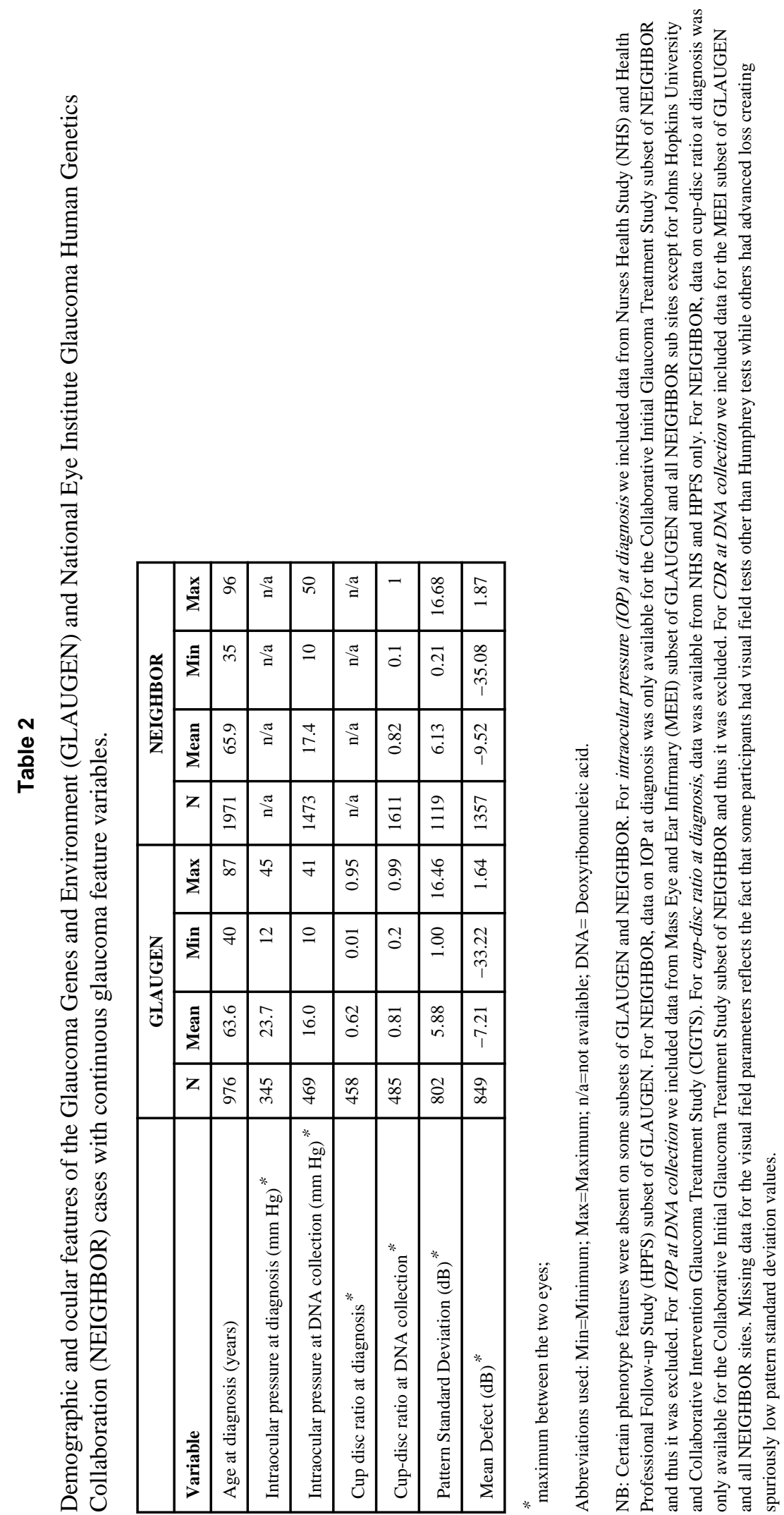
Table 3

Demographic and ocular features of Glaucoma Genes and Environment (GLAUGEN) and National Eye Institute Glaucoma Human Genetics Collaboration (NEIGHBOR) cases with binary glaucoma feature variables.

\begin{tabular}{|l|r|r|r|r|}
\hline & \multicolumn{2}{|c|}{ GLAUGEN } & \multicolumn{2}{c|}{ NEIGHBOR } \\
\hline Variable & \% cutoff & N & \% > cutoff & N \\
\hline Age at diagnosis $\geq 65$ years & 49.3 & 976 & 56.5 & 1971 \\
\hline Gender $(\%$ female) & 58.4 & 976 & 52.1 & 1971 \\
\hline Intraocular pressure $\geq 22$ mm Hg at diagnosis & 67.0 & 976 & 79.4 & 1219 \\
\hline Cup-disc ratio $\geq 0.6$ at diagnosis & 69.9 & 458 & n/a & n/a \\
\hline Cup-disc ratio $\geq 0.6$ at DNA collection & 96.3 & 485 & 95.8 & 1611 \\
\hline Laser trabeculoplasty & 28.2 & 976 & 36.8 & 38 \\
\hline Incisional glaucoma surgery & 15.1 & 976 & 55.0 & 40 \\
\hline Pattern standard deviation $\geq 6$ decibels & 39.8 & 802 & 45.4 & 1119 \\
\hline Mean defect $\leq-13$ decibels & 14.6 & 849 & 26.6 & 1357 \\
\hline Peripheral visual field loss only ${ }^{*}$ & 52.1 & 963 & 31.5 & 1423 \\
\hline Paracentral visual field loss only * & 17.9 & 963 & 3.0 & 1423 \\
\hline Both peripheral and paracentral visual field loss & 30.0 & 963 & 65.1 & 1423 \\
\hline Superior visual field loss only ${ }^{*}$ & 38.8 & 963 & 27.6 & 1423 \\
\hline Inferior visual field loss only * & 28.8 & 963 & 50.7 & 1423 \\
\hline Both superior and inferior visual field loss & 963 & 1423 \\
\hline
\end{tabular}

These visual field loss attributes are applied on a per patient basis. For instance, peripheral visual field loss only means that one or both eyes of a patient have peripheral visual field loss and neither has paracentral field loss.

Abbreviations used: DNA= Deoxyribonucleic acid; $\mathrm{n} / \mathrm{a}=$ not available

NB: Certain phenotype features were absent on some subsets of GLAUGEN and NEIGHBOR. We included data on $C D R>0.6$ at diagnosis from the Nurses Health Study (NHS) and Health Professionals Follow-up Study (HPFS) subsets of GLAUGEN. We included data on $C D R>0.6$ at $D N A$ collection from the Mass Eye and Ear Infirmary (MEEI) subset of GLAUGEN and all NEIGHBOR sites. For laser trabeculoplasty and incisional glaucoma surgery we included data from GLAUGEN as well as the Collaborative Initial Glaucoma Treatment Study and Advanced Glaucoma Intervention Study sub sites of NEIGHBOR. Missing data for the visual field parameters (mean defects and pattern standard deviation) solely reflects the fact that some participants had visual field tests other than Humphrey tests. Missing data for parameters related to the pattern of visual loss (e.g. peripheral visual field loss only) occurred for several reasons including visual field loss was confounded from deficits not attributable to glaucoma like age-related macula or tests assessed segments outside the range of the central 24 or 30 degrees. 
4

$\stackrel{0}{=}$

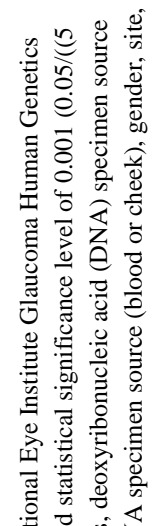

㐘

$\frac{0}{\overparen{a}}$

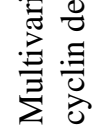

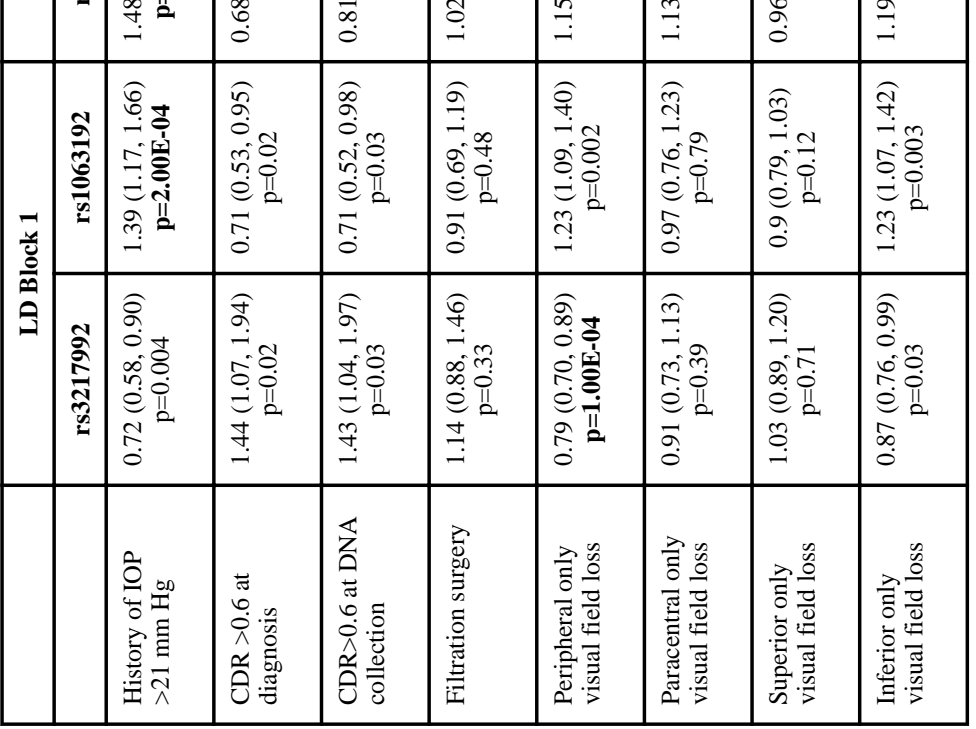

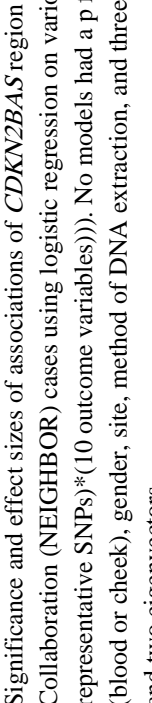

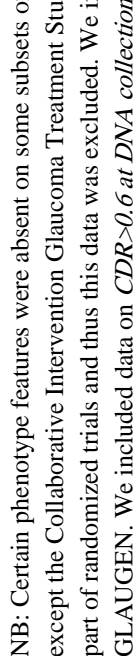

